\title{
A DEPRESSÃO NAS MULHERES DE MEIA-IDADE: ESTUDO SOBRE AS UTENTES DOS CUIDADOS DE SAÚDE PRIMÁRIOS.
}

\author{
Bruno Gonçalves ${ }^{1}$, \\ Teresa Fagulha', \\ Ana Ferreira ${ }^{3}$
}

Resumo: É estudada a prevalência de perturbações depressivas numa amostra de mulheres com idades compreendidas entre os 35 e os 65 anos, utentes dos cuidados de saúde primários. Todas as mulheres $(\mathrm{n}=179)$ responderam à versão portuguesa da Center for Epidemiologic Studies Depression Scale (CES-D) e, as que aceitaram $(n=127)$, participaram numa entrevista clínica estruturada adaptada do módulo de avaliação das perturbações do humor da Structured Clinical Interwiew for DSM-IV Axis I Disorders (SCID-I), a fim de avaliar a presença de várias formas de depressão segundo os critérios do DSM-IV. Verificou-se que 13\% destas mulheres satisfaziam os critérios para o diagnóstico de episódio depressivo major, $19 \%$ satisfaziam os critérios para perturbação distímica e 5,5\% satisfaziam os critérios propostos para episódio depressivo minor. São analisadas as relações da idade, nível de instrução, estatuto conjugal, ter filhos, ter filhos em casa, ter filhos a cargo e outras variáveis sociodemográficas com a ocorrência de perturbações depressivas.

Palavras-chave: mulheres, meia-idade, depressão, cuidados de saúde primários, variáveis sociodemográficas

Depression in midlife woman: A study with users of primary health services (abstract): This study is based on the data collected in an investigation regarding Primary Health Care patients. The prevalence of depressive disorders is studied in a sample of women with ages ranging between 35 and 65 years. All women $(\mathrm{n}=179)$ responded to the Center for Epidemiologic Studies Depression Scale (CES-D), and 127 agreed to participate in a structured clinical interview adapted

\footnotetext{
${ }^{1}$ Professor Associado da Faculdade de Psicologia e de Ciências da Educação da Universidade de Lisboa, Centro de Psicologia Clínica e Experimental. Email: bapg@fpce.ul.pt

2 Professora Associada da Faculdade de Psicologia e de Ciências da Educação da Universidade de Lisboa, Centro de Psicologia Clínica e Experimental.

${ }^{3}$ Professora Auxiliar da Faculdade de Psicologia e de Ciências da Educação da Universidade de Lisboa, LEAD, Centro de Estatística e Aplicações da Universidade de Lisboa, linha de investigação de Análise de Dados Multivariados.
}

PSICOLOGIA, Vol. XIX (1-2), 2005, Edições Colibri, Lisboa, pp. 39-56 
from the evaluation of mood disorders section of the Structured Clinical Interwiew for DSM-IV Axis I Disorders (SCID-I) in order to evaluate the presence of various forms of depression according to the DSM-IV criteria. It was verified that $13 \%$ of these women met the criteria for the diagnosis of a Major Depressive Episode, $19 \%$ met the criteria for Dysthymic Disorder and 5,5\% met the criteria proposed for a Minor Depressive Episode. The relation of age, education level, marital status, work for pay, having children, children at home, dependent children and other socio-demographic variables with the rate of depressive disorders is studied.

\section{Introdução}

A maioria dos autores que se debruçam sobre o tema da meia-idade aponta Jung (1933) como o pioneiro na conceptualização dum período da vida adulta com características particulares que levam a transformações mais ou menos profundas do nosso funcionamento psíquico. Com Elliot Jacques (1965), surge a ideia de crise da meia-idade, crise que é desencadeada pela tomada de consciência da mortalidade, e cuja resolução passa pela reelaboração da posição depressiva infantil. Esta noção de um período crítico de mudança popularizou-se e encontrou eco na análise das alterações sociais e relacionais que acontecem, nas sociedades ocidentais, nas faixas etárias que imediatamente antecedem e sucedem os 50 anos.

Teóricos e investigadores do desenvolvimento (e.g., Colarusso \& Nemiroff, 1981; Erikson, 1950; Levinson, 1979; Vaillant, 1977) têm contestado a ideia de que a vida adulta é, em oposição à infância e adolescência, um período de relativa estabilidade. Se é certo que as mudanças desenvolvimentais são mais nítidas naquelas fases, não é possível negar que o processo de desenvolvimento se estende do nascimento até à morte. Por razões de cariz fundamentalmente social, o estudo da última fase da vida tem conhecido grande desenvolvimento, o qual se traduz no reconhecimento da psicogerontologia como área de estudo e investigação.

$\mathrm{O}$ período da meia-idade tem recebido menos atenção, à excepção, talvez, dos estudos sobre a menopausa, acontecimento que marca a vida da mulher neste período da vida. Simultaneamente, a crescente procura de apoio clínico por pacientes desta faixa etária veio chamar a atenção para a necessidade dum melhor conhecimento das características das experiências de vida que se colocam ao adulto que completou as tarefas do início da vida adulta que frequentemente atingiu um estatuto de sénior, e começa a partilhar o poder (na família e na sociedade) com a geração dos filhos, enquanto lida com as consequências do envelhecimento da geração anterior.

Segundo Levinson (1996), a passagem do início da vida adulta à meia-idade inicia-se com um período de transição, entre os 40 e os 45 anos - uma "ponte desenvolvimental" entre esta era e a anterior -, cuja travessia 
é desencadeada pelo confronto com o fim da juventude e a necessidade de criação duma nova estrutura que comporta o "ainda ser novo", mas estar a envelhecer. Esta nova estrutura vai desenvolver-se entre os 45 e os 50 anos, numa adaptação progressiva à tomada de posição numa outra geração: entre a geração dos filhos que se tornam adultos e a dos pais que entra em declínio. Entre os 50 e os 55 anos, o adulto está a meio da nova era e poderá sentir as mudanças como mais prementes. Se nas fases anteriores não conseguiu realizar as adaptações necessárias para lidar com os novos desafios, poderão surgir crises de desenvolvimento. Da sua resolução depende, quer o modo como no período seguinte, entre os 55 e os 60 anos, conseguirá, ou não, realizar as aspirações e objectivos correspondentes à meia-idade, quer a forma como irá encarar e viver a última fase da sua vida, que começa, então, a desenhar-se no horizonte.

No que diz respeito às mulheres, e se bem que o estudo de Levinson (1996) numa amostra feminina tenha encontrado uma sequência de desenvolvimento idêntica à que verificou no estudo inicial com uma amostra masculina, as alterações relacionais que ocorrem neste período de vida têm nelas um impacto especial, dada a relevância do seu papel na família. Os filhos que crescem e se emancipam, a geração anterior que envelhece (os adultos que cuidaram necessitam agora ser cuidados e a mulher continua a ser a principal cuidadora), as relações conjugais que, por vezes, se desgastaram ou romperam, a menopausa que aponta o final da vida reprodutiva e assinala um marco de envelhecimento (ver artigo de Fagulha \& Gonçalves, neste número), provocam alterações nos esquemas relacionais anteriores e nos papéis sociais que exigem particulares adaptações e reformulações.

Têm surgido vários estudos empíricos sobre este período da vida (e.g., Glazer et al., 2002) em que a mulher está frequentemente confrontada com problemas específicos que podem afectar o seu equilíbrio psíquico, aumentando, nomeadamente, o risco de depressão.

A depressão é, em geral, a patologia psíquica mais frequente, quer quando se considera apenas a depressão major, quer quando se incluem também as formas de sintomatologia menos intensa. Embora muitos sujeitos não procurem ajuda, este facto reflecte-se nomeadamente na frequência com que se encontram problemas depressivos entre os utentes dos cuidados de saúde primários: segundo alguns estudos norte-americanos, entre $6 \% \mathrm{e}$ $10 \%$ destes utentes sofrem de depressão major (Katon, 1987, citado por Volk et al., 1993). Por outro lado, é sabido que esta perturbação afecta predominantemente as mulheres. O rácio entre a prevalência da depressão nas mulheres e nos homens situa-se frequentemente próximo de 2:1 (Noelen-Hoksema, 1990). No entanto, este valor varia consoante as populações estudadas e as formas de depressão consideradas. Num estudo sobre 
a população de Puerto Rico, o rácio relativamente à distimia elevava-se a 4,5:1 (Canino et al., 1987).

Nestas circunstâncias, não será de estranhar que as dificuldades específicas da meia-idade possam eventualmente traduzir-se, em particular no caso da mulher, por um aumento do risco de ocorrência de perturbações depressivas.

$\mathrm{Na}$ verdade, um grande estudo epidemiológico recente, efectuado em vários países europeus (nos quais Portugal não se incluiu), encontrou um ligeiro aumento da depressão major nas mulheres e nos homens da faixa etária 45-54 anos (Angst et al., 2002). Nas faixas etárias superiores, parece haver uma tendência para uma diminuição progressiva da prevalência de depressão major. Mas as variações não são muito marcadas: a prevalência num período de 6 meses para as mulheres de 45-54 anos é de 10,10\%, contra 9,04\% na faixa etária $35-44$ e $8,44 \%$ na faixa $55-64$. Um estudo epidemiológico sobre a prevalência, durante uma semana, de episódios depressivos (severos, moderados ou ligeiros) na população do Reino Unido (Bebington et al., 2003), encontrou resultados próximos. No entanto, neste estudo, o aumento da depressão nas mulheres de 45-54 anos relativamente à faixa etária anterior (35-44 anos) é ainda menos nítido: o que ressalta claramente é a quebra na faixa 55-64 anos relativamente à faixa 16-54 anos considerada globalmente. Pelo contrário, num estudo epidemiológico sobre a população norte-americana, encontrou-se, tal como no estudo europeu referido, um aumento nítido da depressão major nas mulheres de 50-54 anos relativamente às de 45-49 anos (Kessler et al., 1993). Infelizmente, este estudo não incluiu sujeitos com mais de 54 anos. Num outro estudo epidemiológico, igualmente sobre a população norte-americana, encontrou-se apenas uma tendência geral para a diminuição da depressão com a idade, nomeadamente quando se comparam as mulheres com idades entre 18 e 44 anos com as que se situam na faixa dos 45-64 ou com as mais velhas (para uma revisão da literatura, ver Bromberger \& Costello, 1992).

Não parece que as discordâncias entre estes e outros estudos traduzam essencialmente diferenças reais entre as populações estudadas. É provável que resultem, em parte, da forma de avaliação da depressão (entrevista clínica de diagnóstico ou avaliação da sintomatologia depressiva utilizando escalas; Newmann et al., 1996), das faixas etárias incluídas na amostra (abrangendo ou não os sujeitos mais idosos), do critério utilizado (alguns estudos consideram apenas a ocorrência de depressão major, outros utilizam critérios mais latos) e até do tratamento estatístico dos dados. Assim, numa revisão de vários estudos epidemiológicos norte-americanos utilizando a Center for Epidemiologic Studies Depression Scale (CES-D), Kessler e colaboradores (1992) procuraram mostrar que a relação entre a idade e a sintomatologia depressiva não é linear, parecendo haver, tanto 
nas mulheres como nos homens, uma diminuição desta sintomatologia até um mínimo, variável consoante os estudos, e que tende a situar-se próximo dos 55 anos. Depois dessa idade, parece haver de novo uma nítida tendência para o aumento. Este estudo baseia-se nas subescalas que a análise factorial permite definir na CES-D e os resultados não são directamente comparáveis com os dos estudos epidemiológicos inicialmente citados, que se basearam em entrevistas de diagnóstico. No entanto, parece contrariar claramente a ideia de que, na faixa etária dos 45-54 anos, se verificaria um aumento do risco de depressão.

Nestes estudos sobre a população geral, têm sido igualmente estudadas outras características sociodemográficas, como o estado civil ou a situação laboral. Tem sido frequentemente verificado que as mulheres de nível social ou sociocultural mais baixo correm maior risco de depressão (e.g., Gallo et al., 1993; McGrath et al., 1995), embora, neste caso, os resultados não sejam totalmente consistentes (Bromberger \& Costello, 1992). O facto de estar separada ou divorciada parece também aumentar o risco de depressão (e.g., Hirschfeld \& Cross, 1982; Bromberger \& Costello, 1992). Pelo contrário, alguns estudos encontram como resultado que o facto de ter um trabalho remunerado diminui ligeiramente este risco (e.g., Angst et al., 2002), mas Bebbington (1996), fazendo uma revisão da literatura, conclui que o facto de ter um emprego parece ser sobretudo benéfico para as mulheres de nível social mais baixo e que, por outro lado, este efeito é menor no caso das mulheres casadas e com filhos.

A influência do facto de ter filhos parece difícil de avaliar globalmente. Embora haja, pelo menos, um estudo que conclui que o simples facto de ter filhos aumenta o risco de depressão, a maior parte dos autores apontam como factor de risco, sobretudo o facto de ter filhos pequenos a cargo. No entanto, os critérios são variáveis: Bebbington e colaboradores (2003), por exemplo, concluem que o facto de ter filhos com menos de 16 anos em casa constitui um factor de risco. Por outro lado - o que pode parecer algo contraditório - a saída dos filhos de casa tem sido tradicionalmente relacionada com um aumento de risco, embora os dados disponíveis não pareçam confirmar essa ideia (para uma revisão dos principais resultados, cf. Bebington, 1996). Finalmente, Bebbington e colaboradores (2003) observam que a influência de algumas destas variáveis parece variar em função de factores culturais e citam, nomeadamente, os estudos de Mavreas e colaboradores (1986) e de Vázques-Barquero e colaboradores (1987), mostrando que o facto de estar casada parece diminuir o risco de depressão na Europa do Sul muito mais do que na Europa do Norte.

Não dispomos, até à data, de dados epidemiológicos globais relativamente à população portuguesa, ou de estudos com amostras representativas de mulheres utentes dos cuidados de saúde primários em Portugal. 


\section{Objectivos do estudo e população estudada}

O presente estudo teve como objectivos obter uma estimativa quanto à prevalência de perturbações depressivas nas mulheres utentes dos cuidados de saúde primários e estudar as variações eventualmente relacionáveis com o sexo, bem como com a idade, nível de instrução e outras variáveis sociodemográficas.

A população alvo foram as utentes dum Centro de Saúde (C. S. Cascais, nos arredores de Lisboa), com idades compreendidas entre 35 e 65 anos - uma faixa etária em que se situam cerca de $40 \%$ das utentes.

\section{Procedimento e instrumentos utilizados}

Durante um período de 3 meses, todos os utentes com idades compreendidas entre os 35 e 65 anos que tiveram uma consulta com os médicos que colaboraram na investigação foram convidados a participar no estudo. Foi, assim, recolhida uma amostra de 260 sujeitos, dos quais 192 (74\%) eram mulheres. Para cada sujeito, os médicos indicaram quais consideravam ser os problemas de saúde activos.

Numa primeira curta entrevista individual, imediatamente após a consulta médica, cada um dos participantes foi convidado a responder à versão portuguesa da CES-D, anteriormente elaborada pelos dois primeiros autores (Gonçalves e Fagulha, 2003). Foram ainda recolhidos alguns dados demográficos. 179 sujeitos, dos quais 127 (71\%) eram mulheres, aceitaram participar numa segunda entrevista. Esta consistia numa adaptação do módulo de avaliação das perturbações do humor da versão clínica da Structured Clinical Interview for DSM-IV Axis I Disorders (SCID-I; First et al., 1997). O objectivo era ver se se verificavam os critérios para um Episódio Depressivo Major ou para uma Perturbação Distímica. Todos os dados psicológicos considerados relevantes foram igualmente anotados, a fim de permitir a discussão ulterior do diagnóstico. Independentemente do diagnóstico, a avaliação clínica foi ainda utilizada para a construção de um índice de intensidade da sintomatologia depressiva. Este índice foi definido como a soma de todos os sintomas considerados para o diagnóstico de Episódio Depressivo Major cotados segundo uma escala com três graus: ausente, presente mas não satisfazendo o critério do DSM, satisfazendo o critério do DSM. Os sintomas considerados para o diagnóstico de Distimia receberam uma ponderação de 0,75 , dado que correspondem a manifestações menos intensas.

As entrevistas foram realizadas nas instalações do Centro de Saúde, por 5 psicólogos clínicos previamente treinados. Inicialmente, foram reali- 
zadas algumas entrevistas com a presença de um segundo psicólogo, além do entrevistador, a fim de garantir a homogeneidade de critérios. Durante todo o processo, as dúvidas de interpretação dos critérios foram discutidas com os autores.

\section{Características da amostra}

Verificou-se que a amostra era constituída predominantemente por mulheres, o que está de acordo com dados gerais sobre os utentes dos Centros de Saúde em Portugal (Jordão, 1995), bem como noutros países. O Quadro 1 apresenta, sinteticamente, as principais características sóciodemográficas das mulheres que compareceram à entrevista de avaliação clínica $(\mathrm{n}=127)$. As idades destas mulheres distribuíam-se dentro do intervalo considerado de $51,4 \pm 8,9$ anos $(\mathrm{M} \pm \mathrm{DP})$ com uma certa preponderância da faixa etária acima dos 54 anos.

Quadro 1: Características da amostra

\begin{tabular}{cccc}
\hline Idade & Estado civil & Escolaridade & Situação laboral \\
\hline $35-44: 21 \%$ & solteira: $14 \%$ & $<4$ anos: $12 \%$ & empregada: $57 \%$ \\
$45-54: 35 \%$ & casada: $65 \%$ & $4 .^{\circ}$ ano: $31 \%$ & desempregada: $13 \%$ \\
$55-65: 43 \%$ & separadaldivorc.: $16 \%$ & $6{ }^{\circ}$ ano: $7 \%$ & reformada: $12 \%$ \\
& viúva: $6 \%$ & $9 .^{\circ}$ ano: $: 22 \%$ & baixa médica: $5 \%$ \\
& & $12 .{ }^{\circ}$ ano: $17 \%$ & \\
& Licenc.: $10 \%$ & \\
\hline
\end{tabular}

\begin{tabular}{cc}
\hline Profissão* & Filhos \\
\hline (9) não qualificadas: $32 \%$ & sem filhos: $16 \%$ \\
(7) operárias: $7 \%:$ & com filhos em casa, com filhos a cargo: $44 \%$ \\
(5) serviços e vendedoras: $13 \%$ & com filhos em casa, sem filhos a cargo: $12 \%$ \\
(4) pessoal administrativo: $17 \%$ & com filhos, sem filhos em casa ou a cargo: $28 \%$ \\
(3) técnicas intermédias: $5 \%$ & \\
(2) prof. intelectuais e cientificas: $7 \%$ & \\
(1) quadros superiores: $6 \%$ & \\
outras: $13 \%$ &
\end{tabular}

* Os números entre parêntesis referem-se aos grandes grupos da classificação nacional das profissões apenas abreviadamente designados. 


\section{Resultados}

\section{Prevalência das perturbações depressivas}

A entrevista clínica estruturada permitiu concluir que $13,4 \%$ das mulheres satisfaziam os critérios para um episódio depressivo major no momento da consulta médica. Não satisfaziam esses critérios, mas satisfaziam os critérios de distimia 18,9\%. Finalmente, 5,5\% satisfaziam apenas os critérios propostos pelo DSM-IV para um episódio depressivo minor. Ou seja, 38\% das mulheres sofriam de alguma forma de perturbação depressiva.

A pontuação média obtida na CES-D pelas 127 mulheres que aceitaram participar na entrevista clínica $(20,8 \pm 12,4)$ não é significativamente diferente $(\mathrm{p}=0,49)$ da obtida pelas 65 que não participaram $(22,1 \pm 13,0)$. Também não se encontram diferenças significativas quando se comparam as distribuições das outras características sociodemográficas estudadas.

Os médicos assinalaram a presença de problemas depressivos em $18,2 \%$ das mulheres que compareceram à entrevista $(19,7 \%$ dos sujeitos de ambos os sexos). Os limites e dificuldades do diagnóstico médico foram analisados noutro estudo (Gonçalves \& Fagulha, 2004).

\section{Comparação entre mulheres e homens}

As mulheres constituem, em valores absolutos, a maioria dos sujeitos com perturbações depressivas em qualquer das formas consideradas: o rácio entre as mulheres e os homens com alguma forma de perturbação depressiva é de 3,7:1. No entanto, quando não se consideram os valores brutos mas as percentagens, este rácio desce para 1,5:1 e as populações de mulheres e de homens com alguma forma de perturbação depressiva $\left(37,8 \%\right.$ e $37,8 \%$, respectivamente) revelam-se homogéneas $\left(\chi^{2} ; \mathrm{p}=0,10\right)$. Regista-se, contudo, heterogeneidade na composição dos dois sexos quanto à frequência de perturbações distímicas: $18,9 \%$ nas mulheres contra 7,7\% nos homens $\left(\chi^{2} ; \mathrm{p}=0,061\right)$. Pelo contrário, a percentagem de episódios depressivos major é idêntica nas mulheres e nos homens (13,5\% e 13,4\%, respectivamente).

\section{Variações em função da idade e do nivel de instrução}

Embora tivéssemos excluído da nossa amostra as mulheres com menos de 35 ou com mais de 65 anos, o intervalo considerado ainda é suficientemente amplo para podermos estudar a evolução da depressão em função da idade. Como se pode ver no Quadro 2, a frequência mais elevada de episódios depressivos major ou do total de perturbações depressivas 
corresponde à faixa etária dos 45-54 anos. No entanto, a exploração dos dados sugere que há todo o interesse em ter simultaneamente em conta o nível de instrução. Para esse efeito, classificámos no nível de instrução "baixo" todas as mulheres que tinham menos de 9 anos de escolaridade e no nível "alto" as que tinham 9 ou mais anos de escolaridade, e incluímos os resultados correspondentes no mesmo Quadro 2.

Quadro 2: Perturbações depressivas em função da idade e do nível de instrução

\begin{tabular}{|c|c|c|c|c|c|}
\hline & Idade (anos) & $35-44$ & $45-54$ & $55-65$ & Total \\
\hline \multirow{8}{*}{$\begin{array}{c}\text { Amostra } \\
\text { Total }\end{array}$} & a. Episódios depressivos major & $13,8 \%$ & $15,9 \%$ & $11,1 \%$ & $13,4 \%$ \\
\hline & b. Perturbações distímicas & $20,7 \%$ & $15,9 \%$ & $20,4 \%$ & $18,9 \%$ \\
\hline & c. Episódios depressivos minor & $0,0 \%$ & $11,4 \%$ & $3,7 \%$ & $5,5 \%$ \\
\hline & Perturbações depressivas (total $a+b+c$ ) & $34,5 \%$ & $43,2 \%$ & $35,2 \%$ & $37,8 \%$ \\
\hline & Sem perturbações depressivas & $65,5 \%$ & $56,8 \%$ & $64,8 \%$ & $62,2 \%$ \\
\hline & Média de sintomas depressivos & 4,8 & 5,4 & 4,5 & 4,9 \\
\hline & Média CES-D & 19,6 & 19,8 & 22,3 & 20,8 \\
\hline & Total sujeitos & $n=29$ & $n=44$ & $n=54$ & $n=127$ \\
\hline \multirow{3}{*}{$\begin{array}{c}<9 \text { anos } \\
\text { escola }\end{array}$} & Perturbações depressivas (total) & $71,4 \%$ & $53,8 \%$ & $25,8 \%$ & $42,2 \%$ \\
\hline & Sem perturbações depressivas & $28,6 \%$ & $46,2 \%$ & $74,2 \%$ & $57,8 \%$ \\
\hline & Total sujeitos & $n=7$ & $n=26$ & $n=31$ & $n=64$ \\
\hline \multirow{3}{*}{$\begin{array}{c}\geq 9 \text { anos } \\
\text { escola }\end{array}$} & Perturbações depressivas (total) & $22,7 \%$ & $27,8 \%$ & $47,8 \%$ & $33,3 \%$ \\
\hline & Sem perturbações depressivas & $77,3 \%$ & $72,2 \%$ & $52,2 \%$ & $66,7 \%$ \\
\hline & Total sujeitos & $n=22$ & $n=18$ & $n=23$ & $n=63$ \\
\hline
\end{tabular}

Considerando o total das perturbações depressivas (de qualquer tipo), verifica-se que, para o nível de escolaridade baixo, parece haver uma tendência para a diminuição da ocorrência destas perturbações com a idade $(\mathrm{r}=-0,27 ; \mathrm{p}<0,05)$. Verifica-se precisamente o contrário com as mulheres de nível de instrução mais elevado, onde a ocorrência de perturbações depressivas parece tender a aumentar com a idade $(\mathrm{r}=0,33 ; \mathrm{p}<0,01)$.

Dado o pequeno número de mulheres de baixo nível de instrução na faixa etária dos 35-44 anos, procedeu-se igualmente a uma análise das frequências em função do nível de instrução considerando apenas duas classes etárias, definidas pela mediana (52 anos). Esta idade parece, aliás, corresponder aproximadamente ao ponto em que as curvas de frequência relativas aos dois níveis de instrução se cruzam. E verifica-se uma tendência para que a frequência das perturbações depressivas nas mulheres de nível 
de escolaridade baixo com idade inferior ou igual a 52 anos (61\%) seja mais alta do que nas mulheres de nível de escolaridade elevado (22\%) na mesma faixa etária $\left(\chi^{2} ; \mathrm{p}=0,0013\right)$. Acima dessa idade a relação inverte-se (28\% contra $50 \%)$, embora os resultados sejam apenas tendencialmente significativos $(\mathrm{p}=0,074)$.

O Quadro 3 apresenta o resultado de uma análise de regressão logística, tomando como variável dependente a presença ou ausência de alguma forma de perturbação depressiva. A variável idade foi dicotomizada (menos de 53 anos/53 ou mais). Para interpretar estes resultados é preciso ter presente que o efeito de cada variável é avaliado tendo em conta todas as outras e que, quando está presente um termo relativo à interacção de duas variáveis, o efeito isolado de cada uma dessas variáveis é avaliado para o valor zero da outra. Os valores do odds ratio (OR) inferiores a um indicam que a presença da característica em causa diminui o risco de ocorrência de uma perturbação depressiva. Os valores superiores a um indicam, pelo contrário, um aumento do risco.

Estes resultados mostram que a idade, o nível de escolaridade e a interacção entre estes dois factores contribuem significativamente para explicar a presença de alguma forma de perturbação depressiva. Quando não se têm em conta a interacção, nenhuma das variáveis idade ou escolaridade tem um efeito significativo. O modelo obtido permite classificar correctamente $66,9 \%$ dos casos analisados

Quadro 3: Perturbações depressivas em função da idade e do nível de instrução (análise de regressão logística)

\begin{tabular}{lccc}
\hline Variáveis & Sig.* & OR** & Intervalo de confiança a 95\% para OR \\
\hline Escolaridade & 0,002 & 0,178 & {$[0,060 ; 0,531]$} \\
Idade & 0,010 & 0,249 & {$[0,087 ; 0,713]$} \\
Escolaridade x Idade & 0,001 & 14,566 & {$[2,287 ; 39,035]$} \\
\hline
\end{tabular}

* Significância do coeficiente de Wald **Odds Ratio

\section{Influência doutras variáveis sociodemográficas}

A exploração inicial dos dados sugere que, em geral, parece necessário ter em conta o nível sociocultural ou a idade para analisar a relação das outras variáveis sociodemográficas com a sintomatologia depressiva. Assim, no que diz respeito ao estatuto conjugal, verifica-se que, no subgrupo de mulheres mais novas, as casadas apresentam, em média, mais sintomas depressivos do que as solteiras ou separadas, enquanto que, entre as mais velhas, parece verificar-se o contrário. Analogamente, o facto de ter filhos em casa parece ter um efeito diferente em função do nível de escolaridade: 
as mulheres de nível de escolaridade baixo que têm filhos em casa parecem tender a apresentar mais sintomas depressivos, enquanto que, nas mulheres de nível de escolaridade alto, se verifica aparentemente o contrário. Do mesmo modo, o facto de estar desempregada parece estar ligado a um aumento da sintomatologia depressiva nas mulheres mais novas ou nas mulheres de escolaridade baixa, mas não nas mais velhas ou nas de escolaridade alta. A maior parte destas diferenças não são estatisticamente significativas (ou são apenas tendencialmente significativas) quando se utilizam técnicas univariadas - o que pode decorrer, em parte, do pequeno número de mulheres nalgumas das classes definidas. Além disso, existem múltiplas relações entre as variáveis (por exemplo, entre o estado civil e os filhos), o que dificulta a interpretação.

Para analisar estatisticamente estes dados, procurando ter em conta as diversas variáveis e a sua interacção (do ponto de vista da relação com a ocorrência de perturbações depressivas) e determinar quais poderiam ser as variáveis mais importantes, recorremos a uma regressão logística, utilizando um método descendente stepwise. Tomámos como variável dependente a presença ou ausência de alguma forma de perturbação depressiva. Como variáveis independentes foram introduzidas 8 variáveis categoriais: escolaridade (menos de 9 anos/9 ou mais anos de escolaridade), idade (menos de 53 anos/53 ou mais), estado civil (solteira, casada, separada, viúva), ter ou não ter filhos, ter ou não filhos a cargo, ter ou não filhos em casa, estar ou não empregada, viver ou não com cônjuge ou companheiro. Além disso, foram introduzidos termos relativos à interacção da escolaridade e da idade com cada uma das outras variáveis consideradas.

O resultado final desta análise é apresentado no Quadro 4. O modelo obtido permite classificar correctamente $67,7 \%$ dos casos analisados.

Quadro 4: Perturbações depressivas em função das variáveis sociodemográficas modelo resultante da análise de regressão logística descendente stepwise

\begin{tabular}{lccc}
\hline Variáveis & Sig.* & OR $^{* *}$ & Intervalo de confiança a 95\% para OR \\
\hline Escolaridade & 0,004 & 0,209 & {$[0,071 ; 0,614]$} \\
Filhos & 0,023 & 7,885 & {$[1,332 ; 46,675]$} \\
Filhos em casa & 0,040 & 0,224 & {$[0,054 ; 0,933]$} \\
Escolaridade x Idade & 0,002 & 9,449 & {$[2,287 ; 39,035]$} \\
Filhos x Idade & 0,002 & 0,084 & {$[0,017 ; 0,406]$} \\
Filhos a cargo x Idade & 0,047 & 6,088 & {$[1,026 ; 36,139]$} \\
\hline
\end{tabular}

* Significância do coeficiente de Wald ** Odds Ratio 
Partindo do mesmo modelo, mas tomando como variável dependente a obtenção de um resultado na CES-D igual ou superior a 20, a ordem de grandeza dos OR obtidos é semelhante, mas o efeito de algumas das variáveis deixa de ser significativo (Filhos a cargo $x$ Idade) ou é apenas tendencialmente significativo (Filhos $x$ Idade).

\section{Influência das variáveis relacionadas com a menopausa}

A relação entre o estatuto de menopausa e a depressão foi analisada noutro estudo baseado na mesma amostra (Fagulha \& Gonçalves, nesta revista). Verificou-se que o estatuto de menopausa e também as interacções entre o estatuto de menopausa e o nível de escolaridade e entre o estatuto de menopausa e a idade (considerada aqui como variável contínua) contribuem significativamente para a explicação da variável depressão. Essas três variáveis foram acrescentadas ao modelo apresentado no Quadro 3 e procedeu-se a uma análise de regressão logística com um método descendente stepwise, a fim de determinar quais as variáveis que melhor explicavam a variável dependente. No modelo resultante mantêm-se todas as variáveis do modelo inicial e as variáveis relativas ao estatuto de menopausa e à interacção entre a menopausa e a idade, não se revelando significativa apenas a interacção entre o estatuto de menopausa e a escolaridade. Este modelo permite classificar correctamente $70,9 \%$ dos casos analisados.

Utilizou-se o mesmo procedimento partindo do modelo mais complexo apresentado no Quadro 4. O resultado, apresentado no Quadro 5, é análogo: apenas a interacção entre o estatuto de menopausa e a escolaridade não se revela significativa. Este modelo permite classificar correctamente $71,7 \%$ dos casos analisados

\section{Discussão}

Encontrámos uma percentagem de cerca de 38\% de mulheres sofrendo de alguma forma de perturbação depressiva. Este resultado refere-se às mulheres que aceitaram participar na entrevista clínica, mas a distribuição das respostas à CES-D sugere que as que não aceitaram tinham características semelhantes. Temos, no entanto, de ser prudentes quanto à possibilidade de generalizar estes resultados para a população-alvo. Com efeito, os médicos assinalaram a presença de problemas depressivos em 19,7\% dos sujeitos (de ambos os sexos), enquanto que, num grande estudo anterior sobre os utentes dos cuidados de saúde primários (Jordão, 1995), o valor correspondente era de $12,5 \%$. Infelizmente, Jordão não fornece indicações específicas sobre as mulheres do grupo etário considerado: uma 
extrapolação grosseira, a partir do rácio global homens/mulheres, levaria a um valor de cerca de $17 \%$, bastante próximo do observado na presente investigação. Por outro lado, podemos supor que os médicos que aceitaram participar teriam, à partida, uma maior sensibilidade e interesse por este problema e que o conhecimento dos objectivos da investigação possa também ter levado a assinalar mais facilmente a presença de problemas depressivos. No entanto, não podemos excluir a hipótese de que a nossa amostra tenha, de facto, uma percentagem de perturbações depressivas superior aos dados de referência.

Quadro 5: Perturbações depressivas em função das variáveis sociodemográficas e das variáveis relativas à menopausa: modelo resultante da análise de regressão logística descendente stepwise

\begin{tabular}{lccc}
\hline Variáveis & Sig.* & OR** $^{*}$ & Intervalo de confiança a 95\% para OR \\
\hline Escolaridade & 0,007 & 0,203 & {$[0,064 ; 0,643]$} \\
Filhos & 0,014 & 12,442 & {$[1,674 ; 92,480]$} \\
Filhos em casa & 0,028 & 0,177 & {$[0,038 ; 0,827]$} \\
Escolaridade x Idade & 0,004 & 9,785 & {$[2,069 ; 46,283]$} \\
Filhos x Idade & 0,004 & 0,045 & {$[0,005 ; 0,374]$} \\
Filhos a cargo x Idade & 0,029 & 8,548 & {$[1,239 ; 58,991]$} \\
Estatuto de menopausa & 0,027 & & \\
Menopausa & 0,272 & 0,003 & {$[0,000 ; 85,027]$} \\
Pós-menopausa & 0,011 & 0,000 & {$[0,000 ; 0,036]$} \\
Idade x Estatuto de Menopausa & 0,035 & & \\
Idade x Menopausa & 0,282 & 1,115 & {$[0,914 ; 1,361]$} \\
Idade x Pós-menopausa & 0,013 & 1,272 & {$[1,052 ; 1,537]$} \\
\hline
\end{tabular}

* Significância do coeficiente de Wald **Odds Ratio

Dito isto, a prevalência de episódios depressivos major observada é apenas ligeiramente superior aos resultados obtidos por outros estudos com utentes dos cuidados de saúde primários, e a diferença poderá resultar do peso relativamente mais elevado de mulheres na nossa amostra. Pelo contrário, a frequência relativa de distimia nas mulheres é particularmente alta e terá de ser confirmada por outros estudos. Em geral, os dados epidemiológicos sobre a população geral indicam que a prevalência de distimia é inferior à prevalência de depressão Major, nas mulheres como nos homens (Smith \& Weissman, 1991). A elevada frequência de distimia na nossa amostra poderá estar relacionada com características culturais, dado que, 
como vimos, há um estudo sobre a população de Porto Rico (Canino et al., 1987) que encontra igualmente valores muito elevados nas mulheres. Nesse estudo, a proporção entre a prevalência de distimia e de depressão major nas mulheres $(7,5 \%$ e $5,8 \%)$ aproxima-se dos resultados da nossa amostra clínica. No entanto, os resultados deste e dos outros estudos epidemiológicos referidos não são directamente comparáveis com os nossos, não só porque se trata de estudos sobre a população geral, mas também porque os valores referidos dizem respeito à prevalência ao longo da vida.

$\mathrm{O}$ facto de a amostra estudada ser maioritariamente feminina torna difícil tirar conclusões quanto à distribuição das perturbações depressivas em função do sexo na população geral. Em percentagem, o rácio entre as mulheres e os homens com alguma forma de perturbação depressiva é nitidamente inferior ao valor de 2:1 habitualmente encontrado na população geral. Os resultados dum estudo com a CES-D (Gonçalves \& Fagulha, 2003) sugerem que este rácio poderá ser ainda mais elevado na população geral portuguesa. Os resultados obtidos na presente investigação poderão decorrer do facto de a percentagem de homens deprimidos entre os que recorrem aos serviços médicos ser bastante mais elevada do que a percentagem na população geral (estimada com base nos resultados da CES-D), enquanto, no caso das mulheres, esta discrepância não é tão nítida. Independentemente doutros factores, esta diferença traduz provavelmente a maior propensão das mulheres a recorrerem aos serviços médicos mesmo quando a sintomatologia presente é relativamente moderada.

No que respeita à variação da frequência das perturbações depressivas em função da idade (nas mulheres), e apesar do efectivo reduzido da amostra, os resultados globais parecem confirmar a existência de um aumento na faixa etária dos 45-54 anos, o que, como vimos, corresponde aos resultados de um grande estudo epidemiológico europeu. No entanto, verifica-se que há uma interacção muito nítida entre a idade e o nível de instrução (Quadro 3). Com efeito, o facto de ter um nível de escolaridade alto diminui o risco de depressão, mas apenas para as mulheres com menos de 53 anos. Para as mulheres mais velhas, verifica-se exactamente o contrário. O efeito do nível de instrução decorre provavelmente do nível sociocultural (de que o nível de instrução é um bom indicador). Podemos supor que, nas mulheres de nível sociocultural mais baixo, o período entre os 35 e os 52 anos é um período relativamente difícil em que se fazem sentir ainda os problemas relacionados com os filhos a cargo, por exemplo. Passada essa idade (excepto justamente para as que ainda têm filhos a cargo), algumas dificuldades da vida ficam mais ou menos resolvidas e a situação global melhora. Pelo contrário, os resultados sugerem que as mulheres de nível sociocultural mais elevado sofrem menos com essas dificuldades (em particular com todas as dificuldades directamente relacionadas com o nível de 
rendimento) e que são eventualmente mais sensíveis aos problemas do envelhecimento, enquanto tal, que começam a fazer-se sentir na década dos 50 .

Esta análise leva-nos a considerar que a ideia duma crise da meia-idade eventualmente relacionável com um aumento das perturbações depressivas na faixa etária dos 45-54 anos não descreve adequadamente a situação, nem nas mulheres de nível sociocultural baixo, nem nas mulheres de nível mais elevado. O efeito da variável idade parece ser bastante diferente nos dois grupos considerados, mas nem num caso nem noutro encontramos um momento de crise claramente circunscrito.

Será, naturalmente, necessário confirmar estes resultados com amostras representativas da população geral. No entanto, podemos formular a hipótese que algumas das contradições nos resultados dos estudos sobre a relação entre idade e depressão poderão decorrer de não ter sido tida em conta a possibilidade desta ou doutras interacções.

Uma das hipóteses alternativas seria que a crise ou as dificuldades da meia-idade na mulher estariam essencialmente relacionadas com a fase da menopausa e não directamente com a idade (a relação entre a depressão e a menopausa foi analisada noutro artigo: Gonçalves \& Fagulha, neste número). Poderia supor-se que o efeito da variável idade e da interacção da idade com o nível de instrução ou com outras variáveis consideradas neste estudo seria apenas um resultado indirecto da menopausa, já que, embora exista uma certa variação de mulher para mulher, idade e menopausa são duas variáveis estatisticamente correlacionadas. No entanto, quando acrescentámos a variável estatuto de menopausa e os termos relativos à interacção desta variável com o nível de instrução e a idade ao modelo apresentado no Quadro 3, as variáveis incluídas nesse modelo continuaram a contribuir significativamente para a depressão (o mesmo, aliás, se verificou quando procedemos analogamente a partir do modelo apresentado no Quadro 4). Ou seja, temos de admitir que a idade e as outras variáveis consideradas contribuem de forma significativa para a ocorrência de perturbações depressivas, mesmo quando se têm em conta as variáveis especificamente relacionadas com a menopausa.

O modelo resultante da análise de regressão logística com um método descendente stepwise (Quadro 4) privilegia sobretudo as variáveis relacionadas com os filhos. Embora o facto de incluir variáveis muito correlacionadas entre si dificulte a interpretação, os resultados parecem coerentes e não são contraditórios com os de outras investigações. Tem sido verificado, por exemplo, que o ter filhos ainda crianças aumenta o risco de depressão. Infelizmente, neste estudo não foi recolhida informação sobre a idade dos filhos. No entanto, verifica-se que o facto de ter filhos aumenta o risco de depressão para as mulheres mais novas e diminui-o para as mulheres mais velhas. Tendo em conta que é legítimo supor uma correlação ele- 
vada entre a idade das mulheres e a idade dos filhos, uma parte deste efeito poderá provavelmente ser atribuído à idade dos filhos. Por outro lado, como vimos, o facto de ter filhos a cargo aumenta o risco de depressão no caso das mulheres mais velhas, mas, em geral, o facto de ter um ou mais filhos em casa diminui o risco de depressão. Encontramos aqui talvez uma confirmação indirecta do efeito do "ninho vazio" - embora entre as mulheres que não têm filhos em casa se incluam, quer as mulheres cujos filhos já saíram de casa, quer as mulheres sem filhos. A complexidade do modelo também parece razoavelmente coerente quando mostra que, para as mulheres mais velhas, o facto de ter filhos é positivo - mas não o facto de ter filhos a cargo. Ficamos com a impressão de que esta complexidade traduz a complexidade da realidade. O efeito global das variáveis apresentadas nos estudos epidemiológicos como factores de risco para a ocorrência de perturbações depressivas corresponde, certamente, muitas vezes, a uma simplificação excessiva cuja eventual validade estatística esconde situações extremamente diversas.

De qualquer modo, consideramos que este modelo tem apenas um valor descritivo, dado que a amostra é relativamente reduzida. Aliás, o ganho na capacidade preditiva (casos correctamente classificados) relativamente ao modelo que inclui apenas a idade e o nível de instrução é pequeno. Em todo o caso, o facto de este modelo não incluir algumas variáveis (nomeadamente as que se referem ao estatuto conjugal) não deve ser interpretado como uma negação da possível contribuição explicativa dessas variáveis, mas antes como consequência das correlações mais ou menos elevadas de todas as características sociodemográficas estudadas. Só com o recurso a uma amostra de maior dimensão será possível proceder a uma análise mais aprofundada de todas as variáveis consideradas. Um dos aspectos mais interessantes dos resultados deste estudo é alertar-nos para a necessidade de ter em conta as eventuais interacções entre estas variáveis, no seu efeito sobre o risco de depressão.

Finalmente, não podemos esquecer-nos que a amostra é constituída por mulheres que têm problemas de saúde mais ou menos graves. Daí decorre, provavelmente, uma percentagem de mulheres reformadas ou de baixa médica superior à que se poderia encontrar numa amostra da população geral. Por outro lado, não sabemos em que medida o facto de ter problemas de saúde afecta de forma diferente as mulheres, em função, por exemplo, do nível de instrução. Será necessário proceder a outros estudos com amostras da população geral para verificar até que ponto os resultados obtidos são generalizáveis. 


\section{Referências}

Angst, J., Gamma, A., Gastpar, M., Lépine, J.-P., Mendlewicz, J., \& Tylee, A. (2002). Gender differences in depression: Epidemiological findings from the European DEPRES I and II studies. European Archives of Psychiatry and Clinical Neuroscience, 252, 201-209.

Bebbington, P. (1996). The origins of sex differences in depressive disorder: Bridging the gap. International Review of Psychiatry, 8, 295-332.

Bebbington, P., Dunn, G., Jenkins, R., Lewis, G., Brugha, T., Farrell, M., \& Meltzer, H. (2003). The influence of age and sex on the prevalence of depressive conditions: Report from National Survey of Psychiatric Morbidity. International Review of Psychiatry, 15, 74-83.

Bromberger, J. T., \& Costello, E. J. (1992). Epidemiology of depression for clinicians. Social Work, 37 (2), 120-126.

Canino, G., Shrout, P., Bravo, M., Stolberg, R., \& Bird, H. (1987). Sex differences and depression in Puerto Rico. Psychology of Women Quarterly, 11, 443-459.

Colarusso, C. A., \& Nemiroff, R. A. (1981). Adult development. Nova Iorque: Plenum.

Erikson, E. H. (1950). Childhood and society. Nova Iorque: W. W. Norton.

Fagulha, T., \& Gonçalves, B. (2004). Assessment of symptoms of menopause and depression: Influence of the education level and other socio-demographic variables. Poster apresentado na VII European Conference on Psychological Assessment.

First, M. B., Spitzer R. L., Gibbon, M., \& Williams J. B. W. (1997). User's guide for the structured clinical interview for DSM-IV axis I disorders - Clinician version ( $S C I D-C V)$. Washington, DC: American Psychiatric Press.

Gallo, J. J., Royall, D. R., \& Anthony, J. C. (1993). Risk factors for the onset of depression in middle age and later life. Soc Psychiatry Psychiatr Epidemiol, 28, 101-108.

Glazer, G., Zeller, R., Delumba, L., Kalinyak, C., Hobfoll, S., Winchell, J., \& Hartman, P. (2002). The Ohio midlife women's study. Health Care for Women International, 23, 612-630.

Gonçalves, B., \& Fagulha, T. (2003). Escala de depressão do centro de estudos epidemiológicos (CES-D). In M. Gonçalves, M. Simões, L. Almeida, \& C. Machado (Eds.), Avaliação psicológica. (Vol. 1, pp. 33-43).

Hirschfeld, R., \& Cross, C. (1982). Epidemiology of affective disorders: Psychosocial risk factors. Archives of General Psychiatry, 39, 39-46.

Jacques, E. (1965). Death and the midlife crisis. International Journal of Psychoanalysis, 46, 502-514.

Jordão, J. (1995). A medicina geral e familiar. Tese de doutoramento não publicada. Lisboa: Universidade de Lisboa.

Jung, C. G. (1933). Modern man in search of a soul. Nova Iorque: Harcourt, Brace.

Kessler, R. C., Foster, C., Webster, P., \& House, J. (1992). The relationship between age and depressive symptoms in two national surveys. Psychology and Aging, 7 (1), 119-126. 
Kessler, R. C., McGonagle, K. A., Swartz, M., Blazer, D. G., \& Nelson, C. B. (1993). Sex and depression in the National Comorbidity Survey I: Lifetime prevalence, chronicity and recurrence. Journal of Affective Disorders, 29, 85$-96$.

Levinson, D. J. (1979). Toward a conception of the adult life course. In N. J. Smelser \& E. H. Erikson (Eds.), Themes of work and love (pp. 265-290). Cambridge: Harvard University Press.

Levinson, D. J. (1996). The season's of a woman's life. Nova Iorque: Ballantine Books.

McGrath, E., Keita, G. P., Strickland, B. R., \& Russo, N. F. (1995). Women and depression. Risk factors and treatment issues. Final Report of the American Psychological Association's National Task Force on Women and Depression. Washington, DC: American Psychological Association.

Newmann, J., Klein, M., Jensen, J., \& Essex, M. (1996). Depressive symptom experiences among older women: A comparison of alternative measurement approaches. Psychology and aging, 11 (1), 112-126.

Smith, A., \& Weissman (1991). The Epidemiology of depressive disorders: National and international perspectives. In J. Feighner \& W. Boyer (Eds.), Diagnosis of depression (pp. 17-30). Chichester: John Willey.

Vaillant, G. E. (1977). Adaptation to life. Cambridge, MA: Harvard University Press.

Volk, R. J., Pace, T. R., \& Parchman, M. L. (1993). Screening for depression in primary care patients: Dimensionality of the short form of the Beck Depression Inventory. Psychological Assessment, 5 (2), 173-181. 